\title{
Another New Esophagitis: The Lymphocyte Gets Its Turn
}

\author{
David A. Katzka ${ }^{1}$
}

Published online: 1 August 2016

(c) Springer Science+Business Media New York 2016

Establishing the existence of a new disease can start in many ways, for example, an outbreak of an infectious disease with unique clinical characteristics may eventuate in the identification of a new viral or bacterial pathogen. A genome-wide association study (GWAS) may identify a functionally important but abnormal gene associated with an unusual phenotypic presentation of a new disease or a common disease variant. Similarly, a new pathologic finding may provide an essential clue enabling the identification of a novel disease process. For this last category, the difficulty comes in deciding whether the pathologic abnormality is a nonspecific response to multiple disease processes or a singular finding in association with a distinct pathophysiologic process and clinical presentation. Such has been the case with lymphocytic esophagitis.

The existing literature addressing lymphocytic esophagitis has been diffuse, postulating possible associations with Crohn's disease, gastroesophageal reflux, eosinophilic esophagitis, celiac sprue [1], lichen planus, and common variable immunodeficiency. Other reports have attempted to establish lymphocytic esophagitis as a distinct entity. A large part of the problem has been the nonspecific nature of lymphocytes in inflammatory disease. Indeed, disorders as dissimilar as IgG4 disease and celiac sprue are both characterized by dense lymphocytic tissue infiltrates. As a consequence, specificity can only be achieved by identifying the specific lymphocyte types comprising the inflammatory infiltrate, by associating the lymphocytic infiltrate with other histologic findings, and by establishing

David A. Katzka

Katzka.david@mayo.edu

1 Division of Gastroenterology and Hepatology, Mayo Clinic, 200 First Ave., S.W., Rochester, MN 55905, USA clear associations with other specific disease markers that in aggregate support the presence of a particular disease entity.

Although specific markers have yet to be identified for lymphocytic esophagitis, a clearer picture of this entity is beginning to emerge. For example, dysphagia has been reported as a primary symptom of lymphocytic esophagitis in several reports, occurring in over half of diagnosed patients [1-3]. Endoscopically, a normal-appearing esophageal mucosa or an appearance similar to eosinophilic esophagitis (EoE) with plaques, furrows, rings, and strictures has been described [3]. Perhaps as a result, the authors of a recent report hypothesized that lymphocytic esophagitis could represent a "burnt-out" form of EoE [4]. Others have tried to more carefully characterize the pathology of lymphocytic esophagitis. For example, Haque and Genta have described patchy, predominantly peripapillary CD3 + and CD8 + $\mathrm{T}$ cell lymphocytic infiltrates in the absence of other inflammatory cells such as neutrophils or eosinophils. Spongiosis (intercellular epithelial edema) was also a predominant architectural component of the diagnosis [3]. Although these bits and pieces are potentially important clues, they by no means provide a unifying diagnosis for lymphocytic esophagitis.

In this issue of Digestive Diseases and Sciences, Pasricha and colleagues provide a comprehensive overview of 27 patients diagnosed with lymphocytic esophagitis [5]. They defined the disease by a lymphocyte-predominant inflammatory infiltrate devoid of neutrophils and eosinophils. With this definition and a retrospective review of pathology extending to 2004, several characteristics of the disease were described further supporting prior case reports and series. For example, although dysphagia was the most common initial symptom, a variety of EoE or gastroesophageal reflux disease (GERD)-like endoscopic features 
could be present. Stricture location could be anywhere in the esophagus including a pan-esophageal distribution. Novel, however, is the report of finding this entity more commonly found in non-whites and in cigarette smokers when compared to other esophageal diseases such as GERD and EoE. Also, important was the more precise description of lymphocyte count with the authors using $>20$ lymphocytes per high-power field as the diagnostic threshold. At the very least, this important study provides a clearer characterization of this disease with one of the largest case series published. Which one of these descriptive features will stand the test of time, however, remains to be determined.

Do these findings provide a pellucid description of lymphocytic esophagitis? Not yet. When a disease is defined by a series of threshold or descriptive criteria, diagnostic laxity occurs. As a result, those patients (typically a majority) who do not meet all of the criteria but may represent a forme fruste of the disease go undiagnosed and likely untreated. Without a "gold standard" consisting of unambiguous criteria or a distinct pathophysiology, there still remains the risk that multiple and distinct disease processes may manifest as common clinical criteria. For example, although amyloid deposits were considered a pathognomonic finding of systemic amyloidosis, amyloid in tissue serves as a marker of diseases as diverse as Alzheimer's disease (in the brain), diabetes mellitus (in the pancreas), and medullary carcinoma of the thyroid. Another sizable gap in understanding lymphocytic esophagitis is determining effective treatment, which is not only beneficial to the patient but also helpful in defining a disease process where the pharmacology defines a specific pathophysiologic mechanism. Unfortunately, in lymphocytic esophagitis, there are only brief anecdotal mentions of the use of proton pump inhibitors (PPIs) and even antitumor necrosis factor therapy [6] with some success.

Although current knowledge of common gastrointestinal diseases such as GERD or inflammatory bowel disease appears formidable, this understanding results from a series of innumerable small and large breakthroughs occurring over decades or even centuries of study. With a new disease, such as lymphocytic esophagitis, this journey is just beginning. Accumulation and thorough study of this disease such as in the present study contribute greatly to this process. At the very least and most importantly, the UNC group has given some shape to an aspect of this amorphous entity by likely providing a clearer view of one phenotypic and perhaps mechanistic presentation of lymphocytic esophagitis. Future work is needed to find more specific markers within this description such as lymphocyte subsets, carefully defined cytokine, antibody or cellular pathways, and to identify initiating events. Ideally, an animal disease model is commonly helpful in this regard but in practice difficult to create. Finally, trials of therapy such as PPIs and topical steroids need to be conducted to prevent the more severe manifestations of lymphocytic esophagitis such as ring and stricture formation. Until then, the medical community chips away one lymphocyte at a time.

\section{Compliance with ethical standards}

Conflict of interest David A. Katzka received the research funding from Covidien and a consultant at Adare and Receptors Pharmaceutical.

\section{References}

1. Rubio CA, Sjodahl K, Lagergren J. Lymphocytic esophagitis: a histologic subset of chronic esophagitis. Am J Clin Pathol. 2006;125:432-437.

2. Purdy JK, Appelman HD, Golembeski CP, McKenna BJ. Lymphocytic esophagitis: a chronic or recurring pattern of esophagitis resembling allergic contact dermatitis. Am J Clin Pathol. 2008;130:508-513.

3. Haque S, Genta RM. Lymphocytic oesophagitis: clinicopathological aspects of an emerging condition. Gut. 2012;61:1108-1114.

4. Straumann A, Blanchard C, Radonjic-Hoesli S, et al. A new eosinophilic esophagitis (EoE)-like disease without tissue eosinophilia found in EoE families. Allergy. 2016;71:889-900.

5. Pasricha S, Gupta A, Reed CC, Speck O, Woosley JT, Dellon ES. Lymphocytic esophagitis: an emerging clinicopathologic disease associated with dysphagia. Dig Dis Sci. (Epub ahead of print). doi:10.1007/s10620-016-4230-2.

6. Cohen S, Saxena A, Waljee AK, et al. Lymphocytic esophagitis: a diagnosis of increasing frequency. $J$ Clin Gastroenterol. 2012;46:828-832. 